\title{
SHORT-COMMUNICATION
}

\section{Evaluation of remote cameras for monitoring multiple invasive mammals in New Zealand}

\author{
Victor Anton*, Stephen Hartley and Heiko U. Wittmer \\ School of Biological Sciences, Victoria University of Wellington, PO Box 600, Wellington 6140, New Zealand \\ *Author for correspondence (Email: victor.anton@vuw.ac.nz)
}

Published online: 3 October 2017

\begin{abstract}
Numerous conservation projects in New Zealand aim to reduce populations of invasive mammalian predators to facilitate the recovery of native species. However, results of control efforts are often uncertain due to insufficient monitoring. Remote cameras have the potential to monitor multiple species of invasive mammals. To determine the efficiency of cameras as a multi-species monitoring tool, we compared the detection rates of remote cameras and tracking tunnels over 4 non-consecutive days across 40 sites in Wellington. On average, cameras detected significantly more hedgehogs (Erinaceus europaeus) and rats (Rattus spp.) than tracking tunnels, and their images could be used to identify rats to the species level in 50\% of detections. Cameras also detected more possums (Trichosurus vulpecula) but missed recording mice (Mus musculus) on some occasions where tracking tunnels detected them, and vice-versa. We conclude that remote cameras are well-suited for simultaneously monitoring multiple species of invasive mammals in New Zealand.
\end{abstract}

Keywords: invasive species; mammalian pests; restoration; trail cameras; urban ecology

\section{Introduction}

The number of projects aiming to conserve native biodiversity in New Zealand has increased considerably over recent decades (Norton etal.2016). However, the ecological outcomes of many conservation projects are often uncertain due to insufficient monitoring (e.g. Anton et al. 2015). Conservation managers in New Zealand primarily use traps and poisons to supress the number of invasive species including possums (Trichosurus vulpecula), mustelids (Mustela spp.) and rats (Rattus spp.) to levels that allow for the recovery of native species (Pech $\&$ Maitland 2016). The most efficient approach to reducing numbers of invasive mammals is to simultaneously control multiple species (Pech \& Maitland 2016). Conservation managers rely on monitoring tools that record population changes in multiple species over time to measure the success of invasive species management (Ruffell et al. 2015).

Tracking tunnels baited with peanut butter are the national standard technique in New Zealand for monitoring rodents (Gillies \& Williams 2013). Despite not being a standard monitoring protocol, tracking tunnels have also been used to monitor rodents and other mammals including possums and hedgehogs (Erinaceus europaeus) (Morgan et al. 2009; Carter et al. 2016). Tracking tunnels, using a meat-based bait, have also been used to monitor mustelids (i.e. stoats (Mustela erminea) and weasels (Mustela nivalis); Pickerell et al. 2014). The use of tracking tunnels as a multi-species monitoring tool has several limitations; for example, tracking tunnels do not allow for identification at the species level of mustelids and rats (Morgan et al. 2009). Another drawback that tracking tunnels share with other monitoring techniques is the variable probability of animal detection. The probability of tracking tunnels detecting animals can be biased depending on species interference (which animal gets there first), the bait used, food availability and weather conditions (Pickerell et al.
2014; Carter et al. 2016). Inconsistent detection probabilities are an impediment in comparing relative densities among different species and/or ecosystems (Burge et al. 2017). Due to limitations associated with tracking tunnels and other techniques commonly used (e.g. chew cards and WaxTags ${ }^{\mathcal{C}}$ ), multiple monitoring tools are currently deployed to successfully monitor invasive mammals (Pickerell et al. 2014). However, the use of multiple monitoring tools increases both labour and financial costs associated with monitoring programmes. To maximise the efficiency of invasive species control operations, conservation managers require novel approaches to monitoring mammals at spatial extents and with accuracy levels that allow them to judge the appropriate management intervention (Norton et al. 2016).

Camera trapping, the use of remotely-activated cameras to record animals, is used to provide critical information on the distribution, density and behaviour of many mammals worldwide (e.g. Magle et al. 2014; Allen et al. 2016a, b). To efficiently monitor mammals using remote cameras, appropriate deployment of the cameras is crucial (Nichols et al. 2017). Previous research has suggested that animal detection rates from cameras differ based on the methodology (e.g. baited or unbaited stations, horizontal or vertical camera set up, and camera models), habitat, animal traits (e.g. body size and speed) and environmental conditions (Rowcliffe et al. 2011; Nichols et al. 2017). In New Zealand, remote cameras have successfully been used to monitor feral cats (Felis catus) and European rabbits (Oryctolagus cuniculus), as well as interspecific interactions at bait stations (Sam 2011; Latham et al. 2012; Glen et al. 2016).

Compared to other current monitoring methods, cameras allow simultaneous monitoring populations of multiple species without interspecific interferences (Rowcliffe et al. 2011). However, to better understand the benefits and the cost of these devices for monitoring invasive mammals in New Zealand, 
field trials are needed to compare the detection rates from remote cameras with other conventional monitoring methods (Glen et al. 2014).

Our objective was to compare the efficiency (relative sensitivity and specificity) of remote cameras and tracking tunnels for monitoring four common invasive mammals found across New Zealand: possums, hedgehogs, rats, and mice (Mus musculus).

\section{Methods}

We deployed passive infrared triggered cameras (Bushnell, model: 119537$)$ and plastic tracking tunnels $(500 \times 100 \times 100$ mm; Connovation Limited, Auckland, New Zealand) at 40 locations in Wellington, New Zealand (Fig. 1). Half of these locations were in forested areas (regenerating native forest) and the other half were in residential areas (i.e. backyards and gardens). We set up the cameras to record three still photographs (8 megapixels) per trigger. According to manufacturer's specifications, the trigger speed of the cameras after being activated by an animal was 0.6 seconds. We set cameras to the highest sensor level, the lowest flash level and a medium shutter speed. We chose a delay of 30 seconds between triggers to avoid collecting an excessive number of additional photos of the same individual and to maximise memory storage. We attached the cameras to trees $50 \mathrm{~cm}$ above the ground and with a $20^{\circ}$ downward tilt (Fig. 2). The field of detection of our cameras was approximately $2.6 \mathrm{~m}^{2}$ at ground level.

We deployed tracking tunnels $1.5 \mathrm{~m}$ in front of the cameras baited with peanut butter in the middle (all other specifications as per Gillies \& Williams 2013) and left them overnight on two occasions (21 April 2014 and 9 May 2016). To estimate how the use of bait influences the detectability of species by remote cameras, we also inserted unbaited tracking cards into the tracking tunnels and recorded photos on two other occasions (26 April 2014 and 15 May 2016). To minimise neophobic responses from the animals, tunnels were left open for at least 14 days before each recording session (Gillies \& Williams 2013).

We examined each tracking card and photograph to identify the animals recorded. When photo quality allowed, we identified rats detected by the cameras to species level (Fig. 3 ). We were unable to identify rats detected by the tracking tunnels to species level. We calculated the number of trapnights that each target animal was present and absent based on information provided by cameras and tracking tunnels. We used generalised linear mixed models to determine the influence of monitoring device (camera vs tunnel) and bait treatment (baited vs unbaited) on the detection of each species. We omitted an interaction term between device and bait treatment because it was never significant $(\mathrm{P}>0.15)$. Our dependent variable was species' presence/absence and our independent

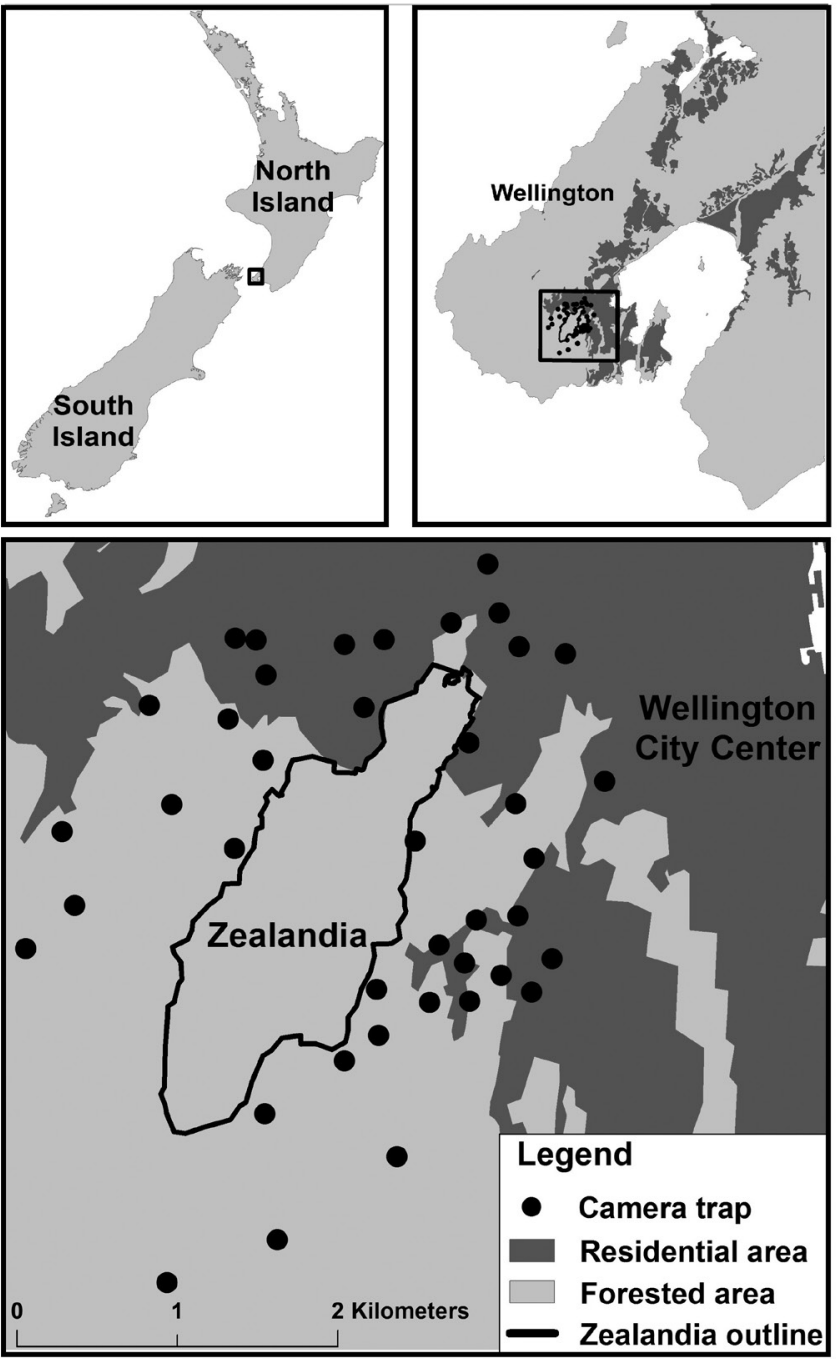

Figure 1. Location of the 40 remote cameras and tracking tunnels in Wellington, New Zealand.
Tracking tunnel

$150 \mathrm{~cm}$
Figure 2. Schematic diagram of the remote camera and tracking tunnel deployment. 


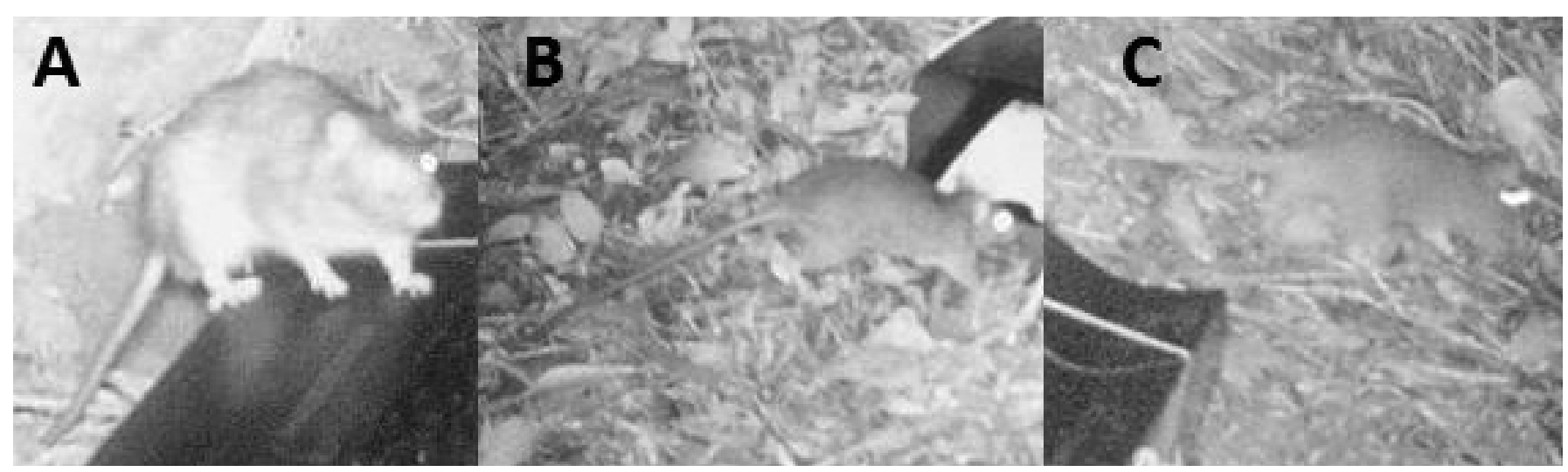

Figure 3. Photographs of (A) Norway rat (Rattus norvegicus), (B) ship rat (Rattus rattus) and (C) a rat we were unable to identify to species level.

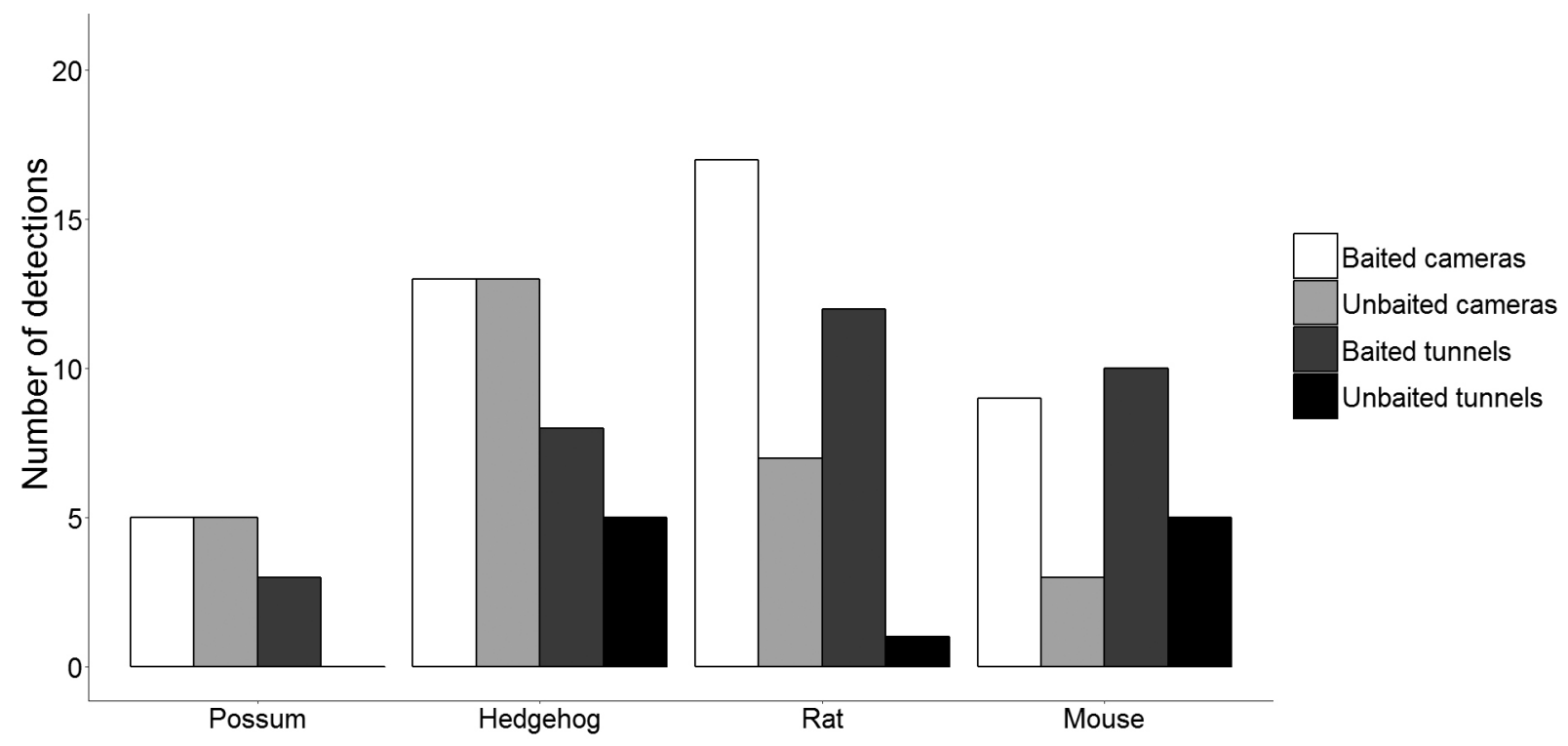

Figure 4. Number of possums, hedgehogs, rats and mice detected on 4 non-consecutive days by remote cameras and tracking tunnels under different bait treatments. Bait treatment included: tracking tunnels baited with peanut butter and unbaited tracking tunnels.

variables were monitoring device and bait treatment. Due to the repeated measures design of our study, we treated site as a random effect (allowing only its intercept to vary) in the model. The analysis was conducted in R (R Core Team 2016) using the lme4 package (Bates et al. 2015). We used $\mathrm{P}<0.05$ as our threshold of significance.

\section{Results}

Remote cameras detected significantly more hedgehogs and rats than tracking tunnels $(\mathrm{P}<0.05)$ (Table 1$)$; indeed, remote cameras recorded all hedgehogs and rats detected by the tracking tunnels (Fig. 4). Tracking tunnels failed to detect some animals recorded by the cameras. For example, tracking tunnels missed recording seven of the ten possums, 13 of the 26 hedgehogs and 11 of the 24 rats detected by cameras (See Appendix S1 in Supplementary Material).

Both tracking tunnels and remote cameras occasionally failed to record mice detected by the other device. Tracking tunnels missed detecting mice during 5 of the 12 trap-nights that cameras recorded mice; but, cameras missed detecting mice during 8 of the 15 trap-nights that tracking tunnels recorded mice (Appendix S1).

The use of peanut butter as bait increased by $363 \%$ and $238 \%$ the detection rates of rats and mice $(\mathrm{P}<0.01$ and $\mathrm{P}<$ 0.05 , respectively) regardless of device (Fig. 4).

We identified rats to species level in 12 of the 24 detections provided by the cameras. Ship rats (Rattus rattus) were detected 11 times while Norway rats (Rattus norvegicus) were detected once.

In addition to our four target species, both devices recorded non-target species during our monitoring period (Table 2).

\section{Discussion}

Our results suggest that remote cameras are more efficient than tracking tunnels for detecting multiple invasive mammals. Remote cameras recorded significantly more hedgehogs and rats than did the tracking tunnels. During some occasions, cameras recorded rats interacting with the tracking tunnels, 
Table 1. Results from generalised linear mixed models used to determine the effect of monitoring device (remote cameras and tracking tunnels) and bait treatment on the detection probability of possums, hedgehogs, rats and mice. A negative coefficient for 'device' indicates that cameras detected more animals than tracking tunnels. A positive coefficient for 'bait' indicates that monitoring devices with bait detected more animals than devices without bait.

\begin{tabular}{llrrr} 
Species & Term & $\begin{array}{r}\text { Coefficient } \\
\text { estimate }\end{array}$ & Std. error & P-value \\
\hline \multirow{2}{*}{ Possum } & Device & -1.35 & 0.69 & 0.052 \\
& Bait & 0.54 & 0.61 & 0.375 \\
\multirow{2}{*}{ Hedgehog } & Device & -0.95 & 0.40 & $0.018^{*}$ \\
& Bait & 0.22 & 0.38 & 0.569 \\
\multirow{2}{*}{ Rat } & Device & -0.79 & 0.39 & $0.044^{*}$ \\
& Bait & 1.56 & 0.44 & $0.000^{*}$ \\
\multirow{2}{*}{ Mouse } & Device & 0.30 & 0.45 & 0.506 \\
& Bait & 1.11 & 0.48 & $0.020^{*}$ \\
\hline
\end{tabular}

but not entering them. Abundance of other food sources and the neophobic character of the animals may have caused the lower detection rates provided by tracking tunnels (Pickerell et al. 2014). Remote cameras were not significantly better at detecting mice. Based on our results we recommend that conservation managers deploy remote cameras if the objective is to simultaneously monitor multiple invasive mammals and/or if high sensitivity to rat presence is required. Tracking tunnels on the other hand should be considered if the objective is to index rodents (rats and mice) in comparison to other monitoring schemes using the national standard method.

Remote cameras and tracking tunnels both missed recording mice on some occasions. We deployed the tracking tunnels and pointed the cameras at the effective detection distance for rats (1.5 m; Rowcliffe et al. 2011). Mice are smaller than rats and often used only one end of the tracking tunnel, impeding their detection by the camera because they were screened by the tracking tunnel. These two factors likely contributed towards the lower mice detection rates recorded by cameras compared to tracking tunnels. As pointed out by Rowcliffe et al. (2011), the effective detection distance of cameras differs for each species. Conservation managers aiming to monitor mice in particular should consider adjusting the effective detection distance for this species when setting up the cameras.

The use of bait in studies with remote cameras to lure animals to the camera's field of view has been widely discussed (e.g. Glen et al. 2013; Rocha et al. 2016). In our study, we detected more target species when baiting the cameras, especially rodents. The use of bait shortens the number of days required to detect species present in an area (Rocha et al. 2016). Bait also facilitates animal identification because it encourages animals to remain in the camera's field of view for longer periods of time (Glen et al. 2013). However, the use of bait violates the assumptions required for determining species richness and accurate density estimates (Rowcliffe et al. 2008). For example, baits are likely to bias the identity and relative numbers of different species recorded by cameras because they favour those species that respond more strongly to the chosen bait (Lazenby et al. 2015). The number of species recorded
Table 2. Species detected by remote cameras and tracking tunnels during 4 non-consecutive nights (two unbaited and two baited with peanut butter) at 40 sites across Wellington City.

\begin{tabular}{lrr} 
Animal & $\begin{array}{r}\text { Remote } \\
\text { cameras }\end{array}$ & $\begin{array}{r}\text { Tracking } \\
\text { tunnels }\end{array}$ \\
\hline Dog (Canis lupus familiaris) & 1 & 0 \\
Possum (Trichosurus vulpecula) & 14 & 3 \\
Cat (Felis catus) & 42 & 0 \\
Rabbit (Oryctolagus cuniculus) & 6 & 0 \\
Hedgehog (Erinaceus europaeus) & 28 & 13 \\
Rat (Rattus spp.) & 24 & 13 \\
Mouse (Mus musculus) & 12 & 15 \\
Blackbird (Turdus merula) & 21 & 0 \\
Dunnock (Prunella modularis) & 3 & 0 \\
Goldfinch (Carduelis carduelis) & 2 & 0 \\
Saddleback (Philesturnus carunculatus) & 1 & 0 \\
Skink (Oligosoma spp.) & 0 &
\end{tabular}

by remote cameras is also likely to be influenced by species interactions at and around the bait (Allen et al. 2016b). Based on our results, monitoring schemes targeting single species or species at low densities might consider baiting the camera traps to minimise trapping effort and costs. However, monitoring schemes aiming for unbiased density estimates of multiple species at medium or high densities should use unbaited camera traps (e.g. Rowcliffe et al. 2011; Rocha et al. 2016).

Cameras detect a greater variety of species than tracking tunnels. In addition to the three mammals commonly targeted with tracking tunnels, remote cameras also recorded more possums and seven other species that tracking tunnels missed completely, including other introduced mammals (especially cats) and native birds. The number of species that could be recorded by remote cameras may be even larger considering that cameras can remain active over much longer periods of time than tunnels without additional effort in the field (Nichols et al. 2017). Longer monitoring periods also enable remote cameras to detect animals at low densities (Rowcliffe et al. 2008). Such information is crucial for understanding complex predator-prey interactions between invasive mammals and the effects of these species on native biodiversity (Pech \& Maitland 2016).

Remote cameras have a number of limitations compared to tracking tunnels. The main limitation is the labour required to classify the footage or photos. However, using public participation (e.g. https://identifyanimals.co.nz) and automatic animal identification software may minimise the time required to collate data provided by cameras (He et al. 2016). The initial capital costs of remote cameras are also considerably higher than those of tracking tunnels (Pickerell et al. 2014). Despite the gradual decrease in equipment costs and the long periods of time that cameras can be active without requiring maintenance, the cost-efficiency of remote cameras compared to more traditional monitoring tools is still low, especially in the short term (Glen et al. 2014). Another limitation is the inability that many commercially available cameras have to detect ectothermic animals, such as lizards and insects, whereas tracking tunnels can detect these species (Jarvie \& Monks 2014). A further drawback of camera trapping is the 
responses that some mammals have shown to the cameras. Remote cameras emit sounds and infrared lights that can be perceived by different species (Meek et al. 2016). However, further research is required to understand the disturbance effects of remote cameras on animals, especially on small- and medium-sized mammals. Other drawbacks associated with cameras include privacy issues, vandalism and theft (Glen et al. 2016), although in our study no such instances occurred.

In our study, we accounted for variation in animal detectability among sites, devices and bait treatment. However, other sources of variation (e.g. weather or species behaviour) might influence the probability of detection of cameras and tracking tunnels (Gillies \& Williams 2013; Allen et al. 2016b; Fancourt 2016). Further research on the interactions among animals, their environment and monitoring devices is required to minimise the number of misdetections (Meek et al. 2016) and to develop new standards. Meanwhile, to ensure correct interpretation of remote camera and tracking tunnel data, we encourage conservation managers to account for imperfect detectability of both monitoring tools (Rowcliffe et al. 2011; Lazenby et al. 2015).

Our results suggest that remote cameras are a robust technique enabling conservation managers to simultaneously monitor multiple invasive mammals in New Zealand (Table 3). The ability of remote cameras to monitor multiple species following pest control operations represents an improvement over current monitoring techniques as it eliminates the need for multiple monitoring devices.

\section{Acknowledgments}

This project would not have been possible without the consent and participation of private landowners and Wellington City Council. We thank Amy Brasch, Kerry Charles, Dannielle Shannahan, Des Smith and an anonymous reviewer for their comments to improve this manuscript, John Innes for confirming our identification of rat species and Victoria University of Wellington's Centre for Biodiversity and Restoration Ecology (CBRE) for covering equipment and vehicle costs.

\section{References}

Allen ML, Wittmer HU, Setiawan E, Jaffe S, MarshallAJ 2016a. Scent marking in Sunda clouded leopards (Neofelis diardi): novel observations close a key gap in understanding felid communication behaviours. Scientific Reports 6: 35433 .

Allen ML, Wilmers CC, Elbroch LM, Golla JM, Wittmer HU 2016b. The importance of motivation, weapons, and foul odors in driving encounter competition in carnivores. Ecology 97: 1905-1912.

Anton V, Hartley S, Wittmer HU 2015. Survival and growth of planted seedlings of three native tree species in urban forest restoration in Wellington, New Zealand. New Zealand Journal of Ecology 39: 170-178.

Bates D, Maechler M, Bolker B, Walker S 2015. Fitting linear mixed-effects models using lme4. Journal of Statistical Software 67: 1-48.

Burge OR, Kelly D, Wilmshurst JM 2017. Interspecies interference and monitoring duration affect detection rates in chew cards. Austral Ecology 42: 522-532.
Table 3. Key advantages of using remote cameras or tracking tunnels as a multispecies monitoring tool.

\begin{tabular}{ll}
$\begin{array}{l}\text { Advantages of remote } \\
\text { cameras }\end{array}$ & $\begin{array}{l}\text { Advantages of tracking } \\
\text { tunnels }\end{array}$ \\
\hline $\begin{array}{l}\text { Detect higher number of } \\
\text { possums, hedgehogs and rats }\end{array}$ & $\begin{array}{l}\text { Can detect endothermic } \\
\text { species such as lizards and } \\
\text { large insects }\end{array}$ \\
$\begin{array}{l}\text { Monitor multiple species } \\
\text { without interspecies }\end{array}$ & $\begin{array}{l}\text { Cost and labour efficient in } \\
\text { the short term }\end{array}$
\end{tabular}

interferences

Detect a broader array of species, including cats, pigs, deer and birds

Privacy issues, vandalism and theft are minor considerations

Enables individual identification of some species (e.g. cats)

Record animal behaviour under natural conditions (trigger may alter behaviour of some species)

Cost and labour efficient in the long term

Carter A, Barr S, Bond C, Paske G, Peters D, van Dam R 2016. Controlling sympatric pest mammal populations in New Zealand with self-resetting, toxicant-free traps: a promising tool for invasive species management. Biological Invasions 18: 1723-1736.

Fancourt BA 2016. Avoiding the subject: the implications of avoidance behaviour for detecting predators. Behavioral Ecology and Sociobiology 70: 1535-1546.

Gillies CA, Williams D 2013. DOC tracking tunnel guide v2.5.2: Using tracking tunnels to monitor rodents and mustelids. Hamilton, Department of Conservation. 14 p.

Glen AS, Cockburn S, Nichols M, Ekanayake J, Warburton B 2013. Optimising camera traps for monitoring small mammals. PLoS ONE 8: e67940.

Glen AS, Warburton B, Cruz J, Coleman M 2014. Comparison of camera traps and kill traps for detecting mammalian predators: a field trial. New Zealand Journal of Zoology 41: $155-160$.

Glen AS, Anderson D, Veltman CJ, Garvey PM, Nichols M 2016. Wildlife detector dogs and camera traps: a comparison of techniques for detecting feral cats. New Zealand Journal of Zoology 43: 127-137.

HeZ, Kays R, Zhang Z, Ning G, Huang C, Han TX, Millspaugh J, Forrester T, McShea W 2016. Visual informatics tools for supporting large-scale collaborative wildlife monitoring with citizen scientists. IEEE Circuits and Systems Magazine 16: 73-86.

Jarvie S, Monks J 2014. Step on it: can footprints from tracking tunnels be used to identify lizard species? New Zealand Journal of Zoology 41: 210-217.

Latham AD, Nugent G, Warburton B 2012. Evaluation of camera traps for monitoring European rabbits before and after control operations in Otago, New Zealand. Wildlife Research 39: 621-628.

Lazenby BT, Mooney NJ, Dickman CR 2015. Detecting species interactions using remote cameras: effects on small mammals of predators, conspecifics, and climate. Ecosphere 6: 1-18. 
Magle SB, Simoni LS, Lehrer EW, Brown JS 2014. Urban predator-prey association: coyote and deer distributions in the Chicago metropolitan area. Urban Ecosystems 17: 875-891.

Meek P, Ballard G, Fleming P, Falzon G 2016. Are we getting the full picture? Animal responses to camera traps and implications for predator studies. Ecology and Evolution 6: 3216-3225.

Morgan DK, Waas JR, Innes JG 2009. An inventory of mammalian pests in a New Zealand city. New Zealand Journal of Zoology 36: 23-33.

Nichols M, Glen AS, Garvey P, Ross J 2017. A comparison of horizontal versus vertical camera placement to detect feral cats and mustelids. New Zealand Journal of Ecology 41: 145-150.

Norton DA, Young LM, Byrom AE, Clarkson BD, Lyver PO, McGlone MS, Waipara NW 2016. How do we restore New Zealand's biological heritage by 2050? Ecological Management \& Restoration 17: 170-179.

Pech R, Maitland M 2016. Conservation of native fauna in highly invaded systems: managing mammalian predators in New Zealand. Restoration Ecology 24: 816-820.

Pickerell GA, O’Donnell CF, Wilson DJ, Seddon PJ 2014. How can we detect introduced mammalian predators in non-forest habitats? A comparison of techniques. New Zealand Journal of Ecology 38: 86-102.

Editorial board member: Des Smith

Received 27 March 2017; accepted 5 July 2017

\section{Supplementary Material}

Additional supporting information may be found in the online version of this article:

Appendix S1. Two-by-two cross classifications of detections and non-detections.

The New Zealand Journal of Ecology provides online supporting information supplied by the authors where this may assist readers. Such materials are peer-reviewed and copy-edited but any issues relating to this information (other than missing files) should be addressed to the authors.
R Core Team 2016. R: a language and environment for statistical computing. R Foundation for Statistical Computing. Version 3.1.2. Vienna, Austria. www.R-project.org.

Rocha DG, Ramalho EE, Magnusson WE 2016. Baiting for carnivores might negatively affect capture rates of prey species in camera-trap studies. Journal of Zoology 300: 205-212.

Rowcliffe JM, Field J, Turvey ST, Carbone C 2008. Estimating animal density using camera traps without the need for individual recognition. Journal of Applied Ecology 45: 1228-1236.

Rowcliffe JM, Carbone C, Jansen PA, Kays R, Kranstauber B 2011. Quantifying the sensitivity of camera traps: an adapted distance sampling approach. Methods in Ecology and Evolution 2: 464-476.

Ruffell J, Innes J, Bishop C, Landers T, Khin J, Didham RK 2015. Using pest monitoring data to inform the location and intensity of invasive-species control in New Zealand. Biological Conservation 191: 640-649.

Sam SA 2011. New monitoring and control tools for simultaneously managing possums, rats and mice in New Zealand. Unpublished PhD thesis, Lincoln, Lincoln University. 149 p. 